\title{
Alcune considerazioni su un caso di sepsi in gastroenterite acuta da Campylobacter jejuni.
}

\section{Dario Cesco,Giorgio Da Rin, Maria Teresa Guidi}

U.O. Microbiologia ULSS 3, Bassano del Grappa(VI)

Key words: campylobacter, enteritis, bacteremia

Campylobacter jejuni gastroenteritis and sepsis: a case report.

\section{SUMMARY}

Campylobacter jejuni is one the most common causes of human diarrea in the world. Complications are relatively rare, but infections have been associated with bacteremia, reactive arthritis and Guillain-Barrè syndrome. We report a $8 \mathrm{I}$-y-old patient with sepsis associated with $\mathrm{CJ}$ enteritis. Difficulties in $\mathrm{CJ}$ detection and identification due to its fastidious nature are also emphasized.

\section{RIASSUNTO}

Campylobacter jejuni (CJ) è una delle più comuni cause di diarrea nel mondo. Le complicazioni sono abbastanza rare e sono state associate con setticemia, artrite reattiva e sindrome di Guillain-Barrè. Di seguito descriviamo un caso di setticemia con i relativi problemi tecnici legati alle difficoltà di crescita e tintoriali di questo microrganismo.

\section{INTRODUZIONE}

Campylobacter jejuni subsp jejuni è un microrganismo ampiamente riconosciuto come un importante agente di gastroenterite infiammatoria acuta ed è la seconda causa più comune dopo Salmonella ${ }^{(3)}$. I sintomi includono febbre, crampi addominali e diarrea con o senza sangue. ${ }^{(2-3)}$

L'infezione è di solito autolimitante e colpisce soprattutto i bambini durante l'estate e inizio autunno. La batteriemia è stata riportata in una media di 1.5 casi su 1000 infezioni intestinali e la percentuale maggiore riguarda i pazienti anziani immunocompromessi. ${ }^{(4)}$ Le complicazioni che possono insorgere nella fase tardiva dell'infezione sono: la sindrome di Guillain-Barrè e l'artrite reattiva. ${ }^{(2)}$

\section{DESCRIZIONE DEL CASO}

Il caso riguarda un paziente di 81 anni, diabetico, colecistectomizzato, epatopatico, ricoverato d'urgenza presso il nostro ospedale per diarrea, vomito, iperpiressia $\left(39^{\circ} \mathrm{C}\right)$, episodio presincopale con breve perdita di coscienza, pallore e sudorazione.

Gli esami ematochimici in entrata rilevavano:

Leucociti $14.11 \times 10^{3} / \mu 1$ con spiccata neutrofilia $89.6 \%$

Piastrine $86 \times 10^{3} / \mu 1$

PCR 6 (v.n. $<0,5)$

$\mathrm{VES}=37 \mathrm{~mm} / \mathrm{h}$

Sono stati eseguiti esami sulle feci:

Esame parassitologico

Ricerca a fresco di uova di elminti: negativa

Ricerca di cisti e trofozoiti di Giardia duodenalis e trofozoiti di Dientamoeba fragilis, (per questi ultimi si è affiancata la colorazione di Giemsa), oocisti di Criptosporidium parvum (colorazione di Kinyoun): negativa

\section{Coprocoltura}

Ricerca Yersinia spp( terreno CIN agar): negativa Ricerca Campylobacter spp(terreno Preston agar): negativa

Ricerca Salmonella spp (terreno MacConkey più arricchimento in selenite broth e semina in SS agar.): negativa

Sono state eseguite due ricerche con sistema immuno-enzimatico(ditta Meridian)

Ricerca tossina A e B di Clostridium difficile : negativa

Ricerca Rotavirus e Adenovirus: negativa

Sono state eseguite anche tre emocolture (sia in anaerobiosi che in aerobiosi) mediante sistema BD Bactec 9240. Due emocolture incubate in aerobiosi si sono positivizzate in terza giornata.

Si è eseguita una subcultura in TSA agar, CNA agar, MacConkey agar, Mannitolo salt agar, Sabouraud agar più uno striscio su cui si è eseguita la colorazione di Gram che evidenziava bacilli Gram negativi debolmente colorati verosimilmente compatibili per Campylobacter spp.

La semina su piastre ha dato i seguenti risultati: Agar cioccolato (BD) incubato in atmosfera di $\mathrm{CO}_{2} 5 \%$ : Crescita di coltura pura (Figura I) Agar sangue (TSA $+5 \%$ sangue di montone $\mathrm{BD}$ ) incubato in atmosfera di $\mathrm{CO}_{2} 5 \%$ : Crescita di coltura pura (Figura II) 
Columbia CNA (BD) incubato in atmosfera di $\mathrm{CO}_{2}$ 5\%: Crescita molto stentata di coltura pura Mannitol Salt (BD) incubato in $\mathrm{O}_{2}$ : Nessuna crescita

Mac Conkey I (BD I) incubato in $\mathrm{O}_{2}$ : Nessuna crescita

Sabouraud GM+C (BD) incubato in $\mathrm{O}_{2}$ : Nessuna crescita

Dalle colonie da agar cioccolato ed TSA agar è stato allestito un vetrino da colorare con il metodo di Gram avendo l'accortezza di usare come controcolorante la fuxina basifenicata non diluita che permette di evidenziare bene la morfologia del Campylobacter.

La lettura del preparato microscopico ha permesso di riconfermare il sospetto diagnostico di sepsi da Campylobacter, avvalorata dalla successiva semina in terreno specifico (BD Campy BF) (Figura 3).

L'identificazione di Campylobacter Jejuni è stata fatta con le seguenti prove biochimiche :

Test dell'ossidasi (BD): Positivo

Test ippurato: Positivo

Sensibilità all' Ac. Nalidixico $(30 \mu \mathrm{g})$ : positiva

Crescita in microaerofilia ed assenza di crescita dopo incubazione in $\mathrm{O}_{2}$

L'antibiogramma non è stato eseguito perchè di solito l'infezione da Campylobacter è di tipo gastroenterico $^{(2-3)}$ autolimitante e non curata con antibiotici.

Nel nostro caso l'antibiogramma sarebbe stato di utilità anche perché antibiotici mirati come $\mathrm{i}$ macrolidi e i fluorochinoloni riconosciuti come mirati per questo tipo d'infezione ${ }^{(2)}$ potevano essere incompatibili con la patologia concomitante come nel nostro caso e per l'insorgenza di resistenza segnalata all'eritromicina ${ }^{(6)}$ Il test di sensibilità andava eseguito in agar sangue con metodo Kirby Bauer.

Inoltre il tentativo di ridurre i tempi della diagnostica microbiologica utilizzando la tecnica dell'antibiogramma diretto sarebbe risultato fuorviante visto che le metodiche automatiche e semi-automatiche a nostra disposizione (Phoenix e Crystal) hanno fallito l'identificazione del patogeno infatti:

Phoenix (BD) con softwaer epicenter (profilo NMIC/ID-55) ha identificato: "Pseudomonas pseudoalcaligens".

BBL Crystal MiND (profilo BBL Crystal Neisseria / Haemophilus 4.0) ha identificato: "Moraxella osloensis" con un fattore di confidenza dello 0.9969.

Il paziente inizialmente è stato messo in terapia

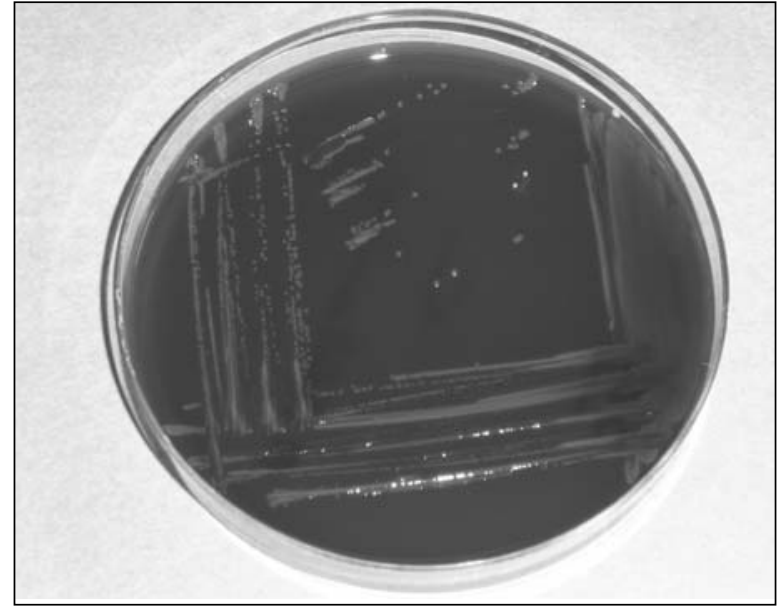

Figura I

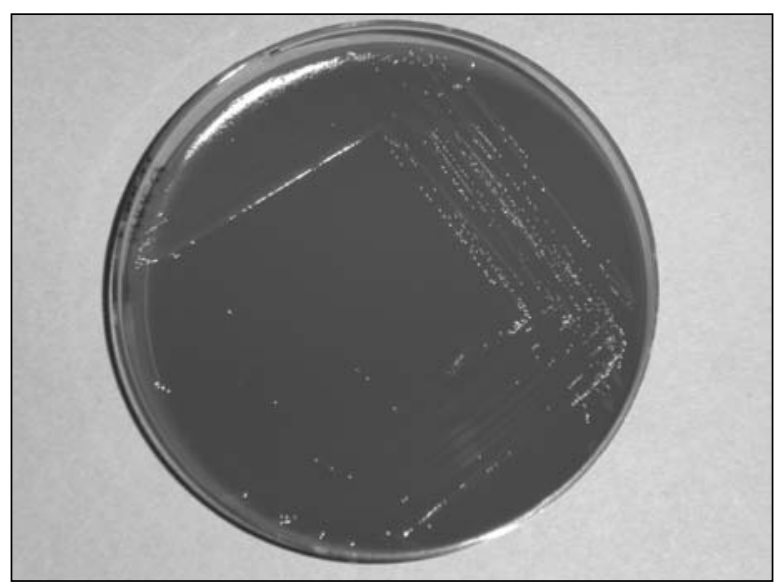

Figura II

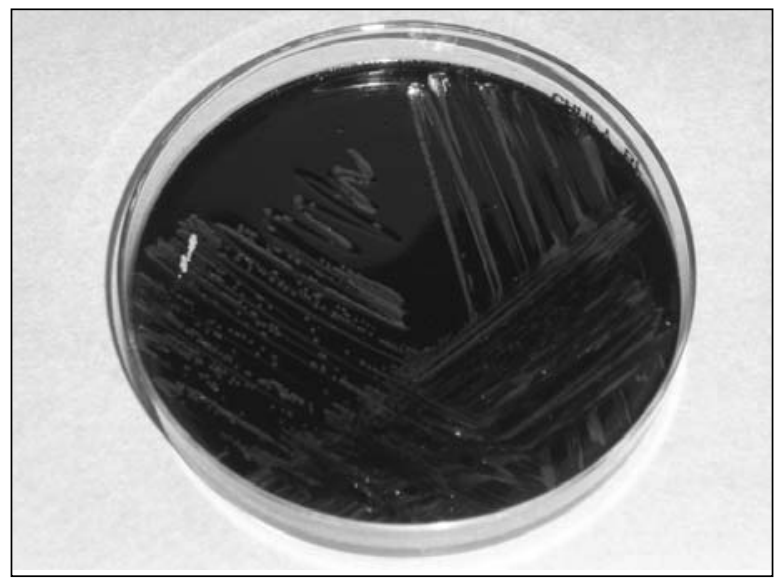

Figura III

con Ceftriaxone, che viene sostituito con Eritromicina $500 \mathrm{mg} 1 \mathrm{x} 4 \mathrm{cps}$ al momento della comunicazione da parte del laboratorio del sospetto di sepsi da Campylobacter; la terapia viene assunta per $10 \mathrm{gg}$., il paziente viene dimesso a guarigione completa. 


\section{CONCLUSIONI}

La nostra conclusione è che questo caso clinico conferma come a tutt'oggi nella microbiologia clinica, l'apporto dell'automazione, non è in grado di sostituirsi all'esperienza e alla professionalità del microbiologo soprattutto in campioni dove la morfologia batterica non è chiara come può essere nel caso in cui Campylobacter si colora debolmente e quindi può portare alla possibilità di false identificazioni e di conseguenza a terapie non mirate.

\section{BIBLIOGRAFIA}

1. Kasten M.J., Allerberger J. P. et al. 1991. Campylobacter bacteraemia: Clinical experience with three different blood culture systems at Mayo Clinic 1984-1990. Infection 19: 376-385.

2. Harrison's principle of internal medicine $16^{\text {th }}$ Edition

3. Nachamkin I. Campylobacter and Arcobacter. pp. 902-914. In: Murray, Baron, Jorgensen, Pfaller and Yolken (Editors). 2003. Manual of Clinical Microbiology. American Society for Microbiology, Washington DC.

4. Skirrov M. B., Jones D. M., Sutcliffe E., Benjamin J. 1993. Campylobacter bacteraemia in England and Walles,1981-1991. Epimediol. Infect. 110: 567-573

5. Wang W.L., Blaser M. J. 1986. Dectention of pathogenic Campylobacter species in blood culture systems. J. Clin. Microbiol 23: 709-714.

6. Giacoboni G. I. Emergency to resistance strains of Campylobacter jejuni isolates from raw retails poultry parts. Analecta Veterin. 2001; 21 1: 63-67 